\title{
Design of Miniaturized Optical Image Stabilization and Autofocusing Camera Module for Cellphones
}

\author{
Yu-Hao Chang, Chieh-Jung Lu, Chien-Sheng Liu, ${ }^{*}$ De-Shin Liu, \\ Shih-Han Chen, Tse-Wei Liao, Wen-Yang Peng, ${ }^{1}$ and Cheng-Hsuan Lin ${ }^{1}$ \\ Department of Mechanical Engineering and Advanced Institute of Manufacturing with High-Tech Innovations, \\ National Chung Cheng University, Chia-yi County 62102, Taiwan \\ ${ }^{1}$ Mechanical and Systems Research Laboratories, Industrial Technology Research Institute, \\ Hsinchu 31040, Taiwan \\ (Received March 31, 2016; accepted May 17, 2017)
}

Keywords: voice coil motor, actuator, camera module, autofocusing, optical image stabilization

For optical image stabilization (OIS) and autofocusing (AF) camera modules, power efficiency and size are two of the most important characteristics. Therefore, in this paper, we propose a novel voice coil motor (VCM) OIS/AF camera module for cellphones to satisfy the requirements of small size and high power efficiency. The design optimization of the proposed VCM OIS/AF camera module is done using simulations. The results of the simulations show that the proposed VCM OIS camera module has excellent characteristics when compared to a conventional VCM OIS camera module.

\section{Introduction}

Autofocusing (AF) adjustment is a very basic function in cellphone camera modules. As smart phones become more common, the demand of consumers for cellphone camera modules is harsher. As a result, more and more cell phone manufacturers have developed new smart phones with optical image stabilization (OIS) camera modules to capture high-quality images. ${ }^{(1)}$ For AF and OIS, the cellphone needs a mini-actuator to generate motion in all three axes. As far as we know, many kinds of mini-actuators (for instance, voice coil motors (VCMs), piezoelectric motors, and stepping motors) are used in cellphone camera modules. ${ }^{(2-4)}$ When compared to other miniactuators, VCMs have the advantage of high-performance dynamic characteristics; therefore, VCMs are the most common in commercial cellphone camera modules. ${ }^{(5)}$

To the best of our knowledge, three mechanisms (prismatic joints of magnetic attracted force structure, flexure-based compliant mechanism, and suspension mechanism) offer a restoring force to balance the Lorentz force $F_{V C M}$ in a three-axis VCM camera module for cellphones. ${ }^{(6-8)}$ Because the conductive suspension mechanism can pass current to the coils to simplify the structure of the camera module and does not have the friction effect, the suspension mechanism is widely used in cellphone camera modules. ${ }^{(9)}$

In this study, we used an industrial microscope (Nikon ECLIPS LV100) to measure the dimensions of a VCM OIS/AF camera module and carried out numerical analysis to accomplish the reverse engineering. From the reverse engineering and from a reference in the patent literature, ${ }^{(9)}$

${ }^{*}$ Corresponding author: e-mail: imecsl@ccu.edu.tw http://dx.doi.org/10.18494/SAM.2017.1599 
we found that the electromagnetic structures of the AF part and the OIS part are independent in a conventional three-axis VCM OIS/AF camera module. The AF and OIS parts have their own coils and magnets, respectively, so the conventional VCM OIS/AF camera module has a complicated structure with many components. In this paper, we propose a new electromagnetic structure in which the AF and OIS parts share the same magnets, so material costs and assembly costs could be effectively reduced.

\section{Design and Principles of Conventional VCM OIS/AF Camera Modules}

Figure 1 illustrates an exploded view of the structure for a conventional VCM OIS/AF camera module. As shown, the conventional VCM OIS/AF camera module can be divided into two parts: the AF part and OIS part. ${ }^{(9)}$ There are four magnets, four coils and one holder in both the AF part and the OIS part; therefore, this design is unfavorable to decrease the assembly cost due to the high number of components. Figures 2(a) and 2(b) show a schematic illustration of the Lorentz forces $F_{V C M-A F}$ and $F_{V C M-O I S}$ in the conventional VCM OIS/AF camera module, respectively. As shown in

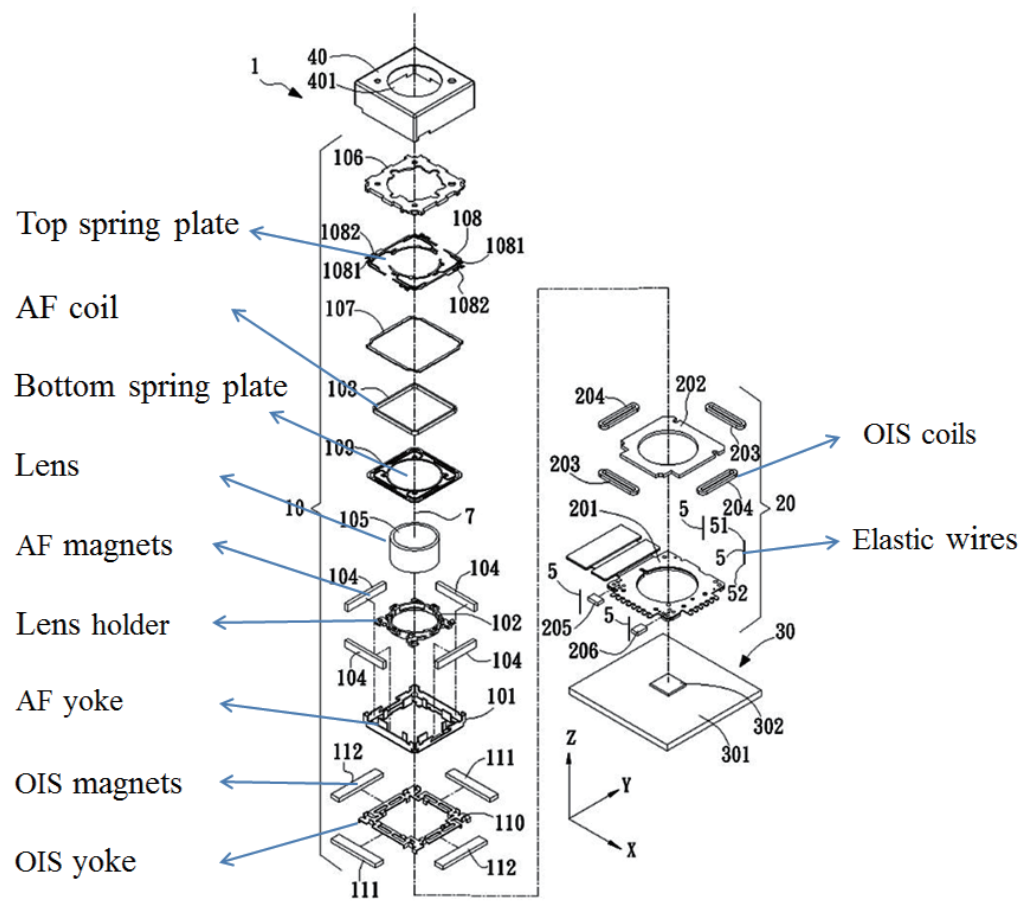

Fig. 1. (Color online) Structure of conventional VCM OIS/AF camera module. ${ }^{(9)}$

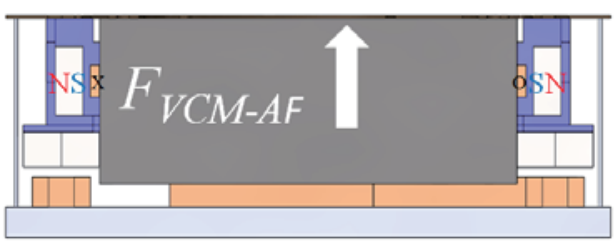

(a)

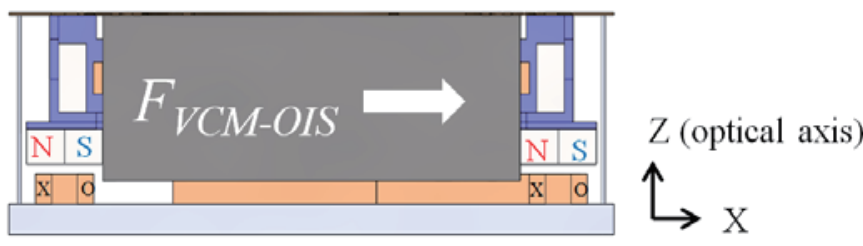

(b)

Fig. 2. (Color online) (a) $F_{V C M-A F}$ and (b) $F_{V C M-O I S}$ of conventional camera module. 
Fig. 2(a), in the AF part, the AF coil, lens holder, and lens are moving parts, while the AF magnets and AF yoke are fixed. When an electric current is passed through the AF coil, the Lorentz force $F_{V C M-A F}$ is generated and the lens is moved along the optical axis ( $Z$ axis) by $F_{V C M-A F}$ to adjust the focus. In addition, there are two spring plates (suspension mechanism, see Fig. 1) that provide a restoring force to balance the $F_{V C M-A F}$. As shown in Fig. 2(b), in the OIS part, the AF part, the OIS yoke, and the OIS magnets are the moving parts, while the OIS coils and base are fixed. When an electric current is passed through the OIS coils, the Lorentz force $F_{V C M-O I S}$ is generated and the lens is moved along the $X-Y$ axis by $F_{V C M-O I S}$ to compensate for the hand shake effect. As in the AF module, four elastic wires (suspension mechanism, see Fig. 1) provide a restoring force to balance the $F_{V C M}$-OIS.

\section{Design and Principles of Proposed VCM OIS/AF Camera Module}

To reduce material and assembly costs effectively, some researchers proposed a modified three-axis VCM OIS/AF camera module in which the AF and OIS parts share the same magnets. ${ }^{(10,11)}$ Referring to the concept of electromagnetic structure in Refs. 10 and 11, we proposed a novel VCM OIS/AF camera module in which two moving parts share the same magnets. Figure 3 illustrates the assembly and the exploded view of the structure for the proposed VCM OIS/AF camera module. The module has four magnets, four AF coils, and four OIS coils. Its $\mathrm{AF}$ and OIS parts share the same magnets, and the yoke is omitted. Due to these two differences between the conventional and proposed VCM OIS/AF camera modules, the proposed VCM OIS/ AF camera module has a simpler electromagnetic structure and lower material costs. As shown in Fig. 4, the magnets of the proposed VCM OIS/AF camera module are axially magnetized (2 pairs of $\mathrm{N}-\mathrm{S}$ poles). The inside of the four magnets provides magnetic flux to AF coils; the underside of the four magnets provides magnetic flux to OIS coils. As in the conventional VCM OIS/AF camera module, two spring plates and four elastic wires provide the restoring forces to balance the $F_{V C M-A F}$ and $F_{V C M-O I S}$, respectively.

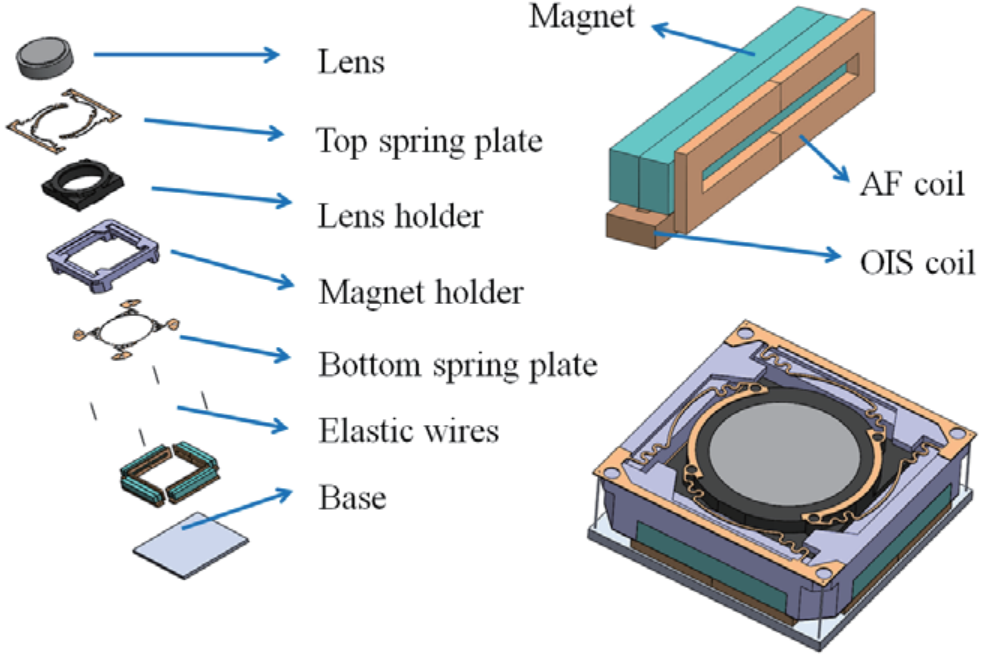

Fig. 3. (Color online) Structure for the proposed VCM OIS/AF camera module. 


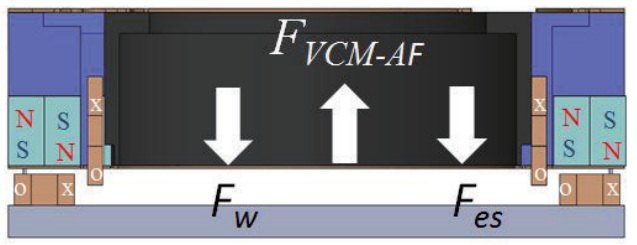

(a)

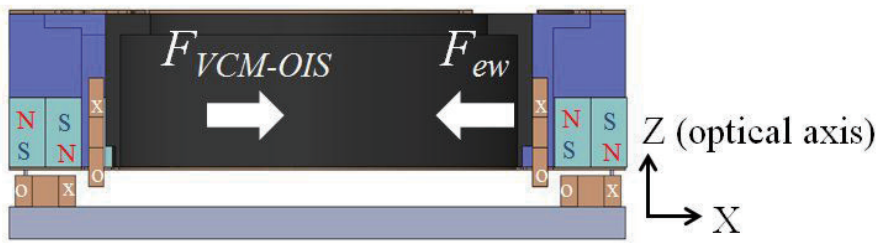

(b)

Fig. 4. (Color online) (a) $F_{V C M-A F}$ and (b) $F_{V C M-O I S}$ of the proposed camera module.

Figures 4(a) and 4(b) show a schematic illustration of the actuation principle of the VCM OIS/ AF camera module. As shown in Fig. 4(a), in the AF part, the spring plates provide an elastic restoring force $F_{e s}$ to keep the lens in the home position at any posture $\left(F_{e s} \geq F_{w}\right)$. When the camera module is in a difficult posture, the Lorentz force $F_{V C M-A F}$ overcomes the weight of the moving part and the elastic restoring force of the spring plates to move the lens to a specific position. The force equilibrium equation can be written as

$$
F_{V C M-A F} \geq F_{e s}+F_{w}
$$

As shown in Fig. 4(b), in the OIS part, when the camera module is in a difficult posture, the Lorentz force $F_{V C M \text {-OIS }}$ overcomes the weight of the moving part and the elastic restoring force of the elastic wires to move the lens to a specific position. The force equilibrium equation can be written as

$$
F_{V C M-O I S} \geq F_{e w}+F_{w}
$$

\section{Simulation of Suspension and Electromagnetic Structure}

To design the VCM OIS/AF camera module, a finite element analysis was carried out using the commercial software ANSYS to analyze the suspension mechanism (spring plates and electric wires), and the main design parameters of the suspension mechanism are shown as Table 1. Figures 5(a)-5(c) show the results of the simulation for the von Mises stresses on the top spring plate (displacement of $0.26 \mathrm{~mm}$ ), the bottom spring plate (displacement of $0.26 \mathrm{~mm}$ ), and elastic wires (displacement of $0.085 \mathrm{~mm}$ ), respectively. The values of the stress on the spring plates and elastic wires were under the value of the yield stresses of the materials. Because the function of the top and bottom springs is to maintain the lens in the correct focusing position during operation, we assigned the maximum operation displacement $(0.26 \mathrm{~mm})$ as a boundary condition for the $\mathrm{AF}$ springs to evaluate the stiffness during operation. Similarly, we assigned the maximum offset value $(0.085 \mathrm{~mm})$ for the OIS wires to evaluate the anti-offset stiffness. The stress distribution results demonstrate that the stiffnesses were strong enough to prevent the springs and wires from being permanently deformed during operation.

To design suitable dimensions for the coils and magnets, a finite element analysis was performed using the commercial software MAGNET. Table 2 lists the main design parameters of the electromagnet structure. Because the AF and OIS parts share the same magnets, the crosssectional areas of both AF coils and OIS coils need to be designed to generate the maximum Lorentz force. Figure 6 illustrates the magnetic flux distribution for the proposed VCM OIS/AF 
Table 1

Design parameters of suspension mechanism.

\begin{tabular}{|c|c|c|}
\hline Variable & Top spring plate and bottom spring plate & Elastic wires \\
\hline Material & C19900HP-GSH & MX215 \\
\hline Elastic Modulus E (GPa) & 127 & 125 \\
\hline Density $\left(\mathrm{g} / \mathrm{cm}^{3}\right)$ & 8.7 & 8.95 \\
\hline Yield Stress (MPa) & 1390 & 820 \\
\hline Tensile Strength, Ultimate (MPa) & $1300-1600$ & $910-1060$ \\
\hline \multirow{2}{*}{ Dimensions $\left(\mathrm{mm}^{3}, \mathrm{~mm}\right)$} & Top spring $\quad 10.24 \times 10.24 \times 0.055$ & $\varnothing=0.03$ \\
\hline & $10.24 \times 10.24 \times 0.04$ & Length $=2.8$ \\
\hline
\end{tabular}

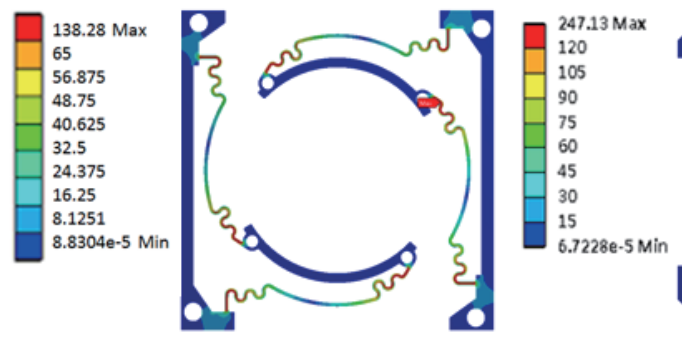

(a)

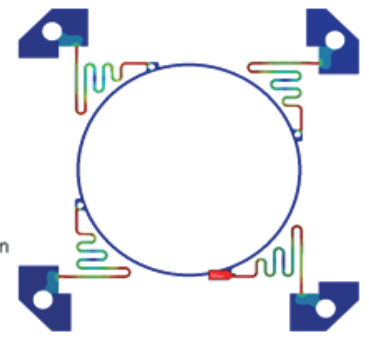

(b)
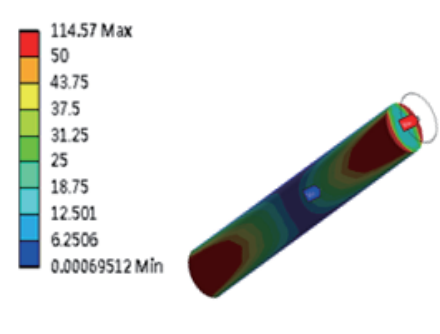

(c)

Fig. 5. (Color online) von Mises stresses of (a) top spring plate (138.25 MPa), (b) bottom spring plate (247.13 $\mathrm{MPa})$, and (c) elastic wires (114.57 MPa).

Table 2

Design parameters of electromagnet structure.

\begin{tabular}{llc}
\hline & Variable & Corresponding value \\
\hline \multirow{3}{*}{ Permanent magnet } & (BH) max (MGOe) & $43-46$ \\
& Material & NdFeB N46H \\
& Dimensions $(\mathrm{mm})$ & $1.2 \times 1 \times 6.6$ \\
\hline \multirow{4}{*}{ AF coils } & Outer dimensions $(\mathrm{mm})$ & $0.56 \times 0.25 \times 6.6$ \\
& Cross-sectional area $(\mathrm{mm})$ & 84 \\
& Winding turns (turns) & 46 \\
\hline \multirow{4}{*}{ OIS coils } & Electrical resistance $(\Omega)$ & $1 \times 0.45 \times 7.16$ \\
& Outer dimensions $(\mathrm{mm})$ & $0.28 \times 0.45$ \\
& Cross-sectional area $(\mathrm{mm})$ & 100 \\
\hline Air gap width between permanent magnet and coil $(\mathrm{mm})$ & 29 \\
\hline Dimensions of camera & Winding turns (turns) & 0.12 \\
\hline
\end{tabular}

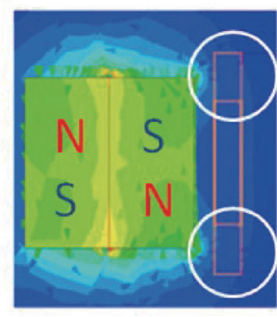

(a)

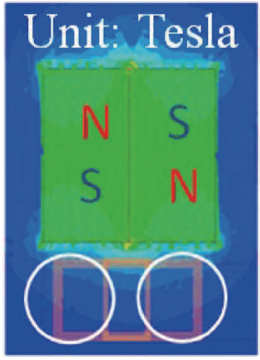

(b)

Fig. 6. (Color online) Magnetic flux distribution: (a) AF part and (b) OIS part. 
camera module. As shown, the areas in white circles have the maximum magnetic flux density, so the AF coils are designed to be longer than the height of the magnets, and the OIS coils are designed to be shorter than the width of the magnets. Meanwhile, the dimension given avoids collisions between the AF coils and OIS coils when they function. Figure 7 shows a comparison between the simulation of Lorentz forces $F_{V C M-A F}$ and $F_{V C M}$-OIS with the displacement as a function of an input current of $30 \mathrm{~mA}$ for the conventional and proposed VCM OIS/AF camera modules. Both the AF and OIS parts of the proposed structure have a larger Lorentz force, indicating that the proposed VCM OIS/AF camera module has a higher power efficiency.

Figure 8(a) presents the results of the simulation of Lorentz force $F_{V C M-A F}$ and the sum of the spring elastic restoring force $F_{e s}$ plus the weight of the moving part $F_{w}$ with the displacement as a function of input currents for the AF part. Figure 8(b) presents the results of the simulation of the Lorentz force $F_{V C M}$-OIS and the sum of the wire elastic restoring force Few plus the weight of the moving part $F_{w}$ with the displacement as a function of input currents for the OIS part. As shown, the starting current for the AF part is $10 \mathrm{~mA}$. A current of $50 \mathrm{~mA}$ is needed to overcome the sum of the $F_{e s}$ plus $F_{w}$ to move the lens $0.085 \mathrm{~mm}$ (the stroke of OIS part).

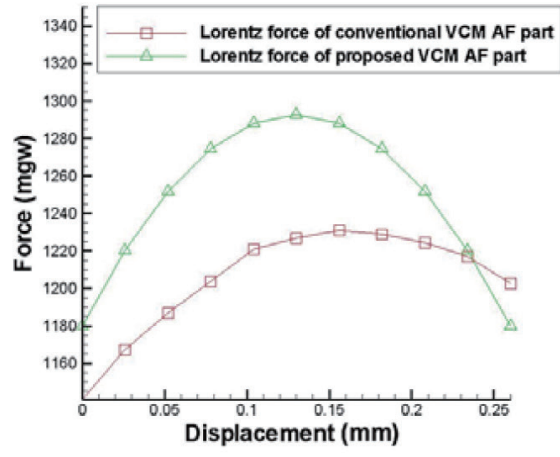

(a)

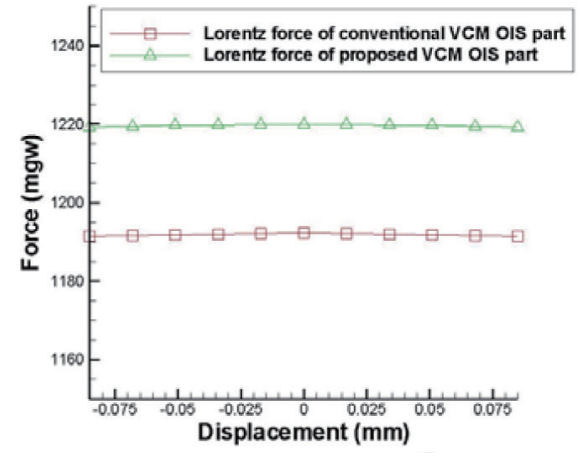

(b)

Fig. 7. (Color online) Comparisons of Lorentz forces $F_{V C M-A F}$ and $F_{V C M-O I S}$ : (a) AF part and (b) OIS part.

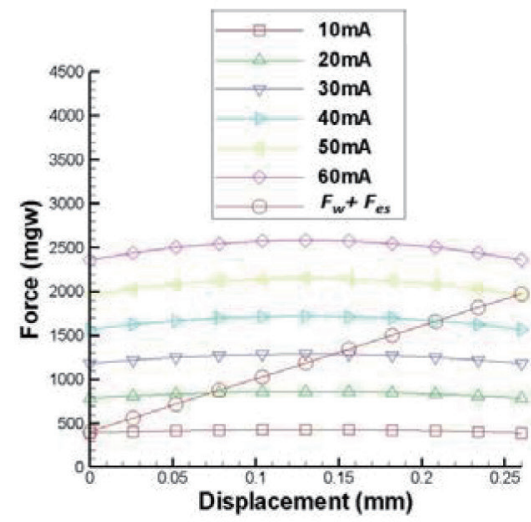

(a)

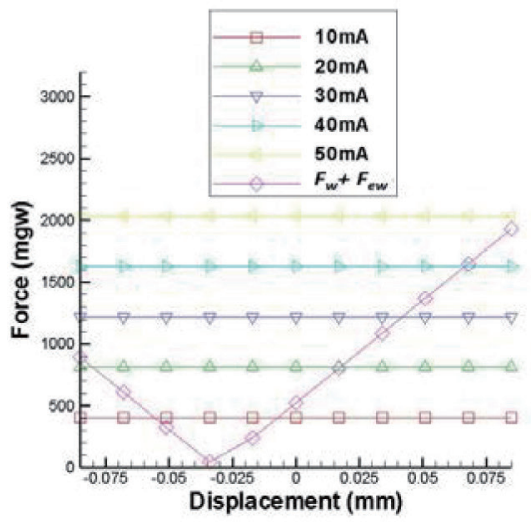

(b)

Fig. 8. (Color online) Simulation results for Lorentz forces and elastic restoring forces: (a) AF part and (b) OIS part. 


\section{Conclusions}

This report has presented a novel VCM OIS/AF camera module for cellphones. Its AF and OIS parts share the same magnets so material and assembly costs may be effectively reduced. Simulations show that the proposed VCM OIS/AF camera module has a smaller input current (10 $\mathrm{mA}$ ) and a lower power consumption compared to the conventional VCM OIS/AF camera module.

\section{Acknowledgments}

This work was supported by the Industrial Technology Research Institute and the Ministry of Science and Technology of Taiwan under Grant Nos. MOST 103-2221-E-194-006-MY3, 1043011-E-194-001，104-2622-E-194-003-CC2，105-2221-E-194-013-MY5，105-2218-E-194-004, 105-2218-E-194-003，106-2218-E-194-002，106-3114-8-194-001，106-2622-E-194-004-CC2, and 106-2622-E-194-005-CC3.

\section{References}

1 R. J. Topliss: US Patent No. 2014/009631 A1 (2014).

2 H. Harada and Y. Tanaka: Sens. Mater. 27 (2015) 309.

3 C. L. Zhang, X. N. Zhang, M. L. Xu, and Y. J. Luo: Int. J. Appl. Electromagn. Mech. 45 (2014) 83.

4 J. S. Choi, J. Yoo, and N.C. Park: Microsyst. Technol. 15 (2009) 1489.

5 C. S. Liu and P. D. Lin: Sens. Mater. 20 (2008) 319.

6 C. S. Liu, Y. U. Cheng, and H. F. Li: Int. J. Appl. Electromagn. Mech. 51 (2016) 61.

7 C. Kim, M. G. Song, Y. J. Kim, N. C, Park, K. S. Park, Y. P. Park, K. S. Shin, J. G. Kim, and G. S. Lee: Microsyst. Technol. 19 (2013) 1633.

8 K. H. Kim, S. Y. Lee, and S. Kim: Opt. Express 17 (2009) 5891.

9 F. Y. Wu, S. T. Huang, and J. J. Lin: US Patent No. 2013/0215511 A1 (2013).

10 M. Sugawara: US Patent No. 2013/0016427 A1 (2013).

11 C. L. Hsieh, H. Y. Wang, Y. H. Chang, and C. S. Liu: Microsyst. Technol. (2017) (in press). 